\title{
A Comparative Study of Cyclic Fatigue of 10 Different Types of Endodontic Instruments: an in Vitro Study
}

\section{Komparativno istraživanje cikličkog zamora deset različitih vrsta endodontskih instrumenata: istraživanje in vitro}

Department of Endodontics and Restorative Dentistry, Catholic University of Valencia, Spain
Zavod za endodonciju i restaurativnu dentalnu medicinu Katoličkog sveučilišta u Valenciji, Španjolska

Abstract

Objective: This study was designed to test the null hypothesis that there were no significant differences between size 25 files F360, F6 SkyTaper, Hyflex EDM, iRace, Neoniti, One Shape Protaper Next, Reciproc, Revo-S and Wave One Gold in terms of resistance to cyclic fatigue and length of broken fragments. Material and methods: 300 new size 25 files of the systems studied were selected $(n=30)$. The instruments were mechanized with a X-Smart Plus endo motor at the speed and torque recommended by the manufacturer, holding the instruments steady with a clamping mechanism, with passive adjustment and without pressure in a stainless-steel block. The time was calculated in seconds until fracture. The number of fatigue cycles was calculated as (Resistance (s) $x$ Speed)/60. The separated fragment lengths were measured with a digital Vernier calliper. A statistical analysis was performed with the SPSS 18 programme at a 95\% confidence level, using the Levene's Test to compare variances, the Welch's Test to compare means, and the Games-Howell's Test to reveal differences between groups. Results: The Levene's Test showed no equal variances $(P<0.05)$. The Welch's Test $(P<0.05)$ showed significant differences in cyclic fatigue and separated fragment lengths. The GamesHowell test $(\mathrm{P}<0.05)$ exhibited significant differences in multiple comparisons $(\mathrm{P}<0.05)$. Conclusions: The systems with CM-Wire (Hyflex EDM and Neoniti) were superior in resistance to the other systems for cyclic fatigue. For separated fragment lengths, F360 (conventional NiTi) and Reciproc (M-Wire) were better significantly better in terms of resistance.
Received: June 30, 2018

Accepted: December 12, 2018

Address for correspondence Jorge Rubio

Catholic University of Valencia, Spain Department of Endodontics and Restorative Dentistry jorrucli@hotmail.com

\section{Introduction}

The use of Nickel-Titanium (NiTi) instruments has increased due to the advantages they present compared to manual files (1-2), which are used in canal-shaping procedures to reduce bacteria levels and facilitate irrigation and obturation for long-term success $(3,4)$. Their main advantages are elasticity and cutting efficiency $(5,6)$, and also the frequent changes of characteristics and cross-section of endodontic files have increased their resistance to cyclic fatigue (7-9), but their major problem is fracturing in root canals $(1,2)$.

With regard to currents systems, new manufacturing processes and alloys such as CM-Wire, M-Wire and Gold-Wire have been developed to improve conventional $\mathrm{NiTi}$ in recent years (10). Another improvement has been a reciprocating motion, an oscillating motion that rotates the files in one direction then reverses their motion before effecting a complete rotation (11). The useful life of instruments can be increased by reciprocating motion, as it presents better resistance to cyclic fatigue than continuous rotation $(12,13)$. Frequency, apical strength of instrument, pressure applied by the operator and enlargement of canal are among the clinical factors
Uvod

Uporaba instrumenata od nikal-titanijeve legure (Ni-Ti) povećala se zbog prednosti koje ti elementi imaju u usporedbi s ručnim iglicama $(1,2)$ koje se koriste u postupcima oblikovanja kanala, smanjenja broja bakterija u njima, za olakšavanje irigacije te punjenje, a u svrhu dugoročnog uspjeha $(3,4)$. Njihove glavne prednosti su elastičnost i učinkovitost pri rezanju $(5,6)$, a stalne promjene svojstava i presjeka endodontskih iglica povećale su njihovu otpornost na ciklički zamor (7 - 9). No, glavni problem i dalje ostaje puknuće instrumenata u kanalu $(1,2)$.

Posljednjih godina novi proizvodni postupci i legure, kao što su CM žica, M žica te zlatna žica (engl. Gold-Wire), poboljšali su konvencionalnu nikal-titanijevu leguru (10). Drugo poboljšanje bilo je uvođenje recipročnog pokreta koji rotira iglicu u jednom smjeru, a zatim je vraća prije nego što se dogodi potpuna rotacija (11). Korisni vijek trajanja instrumenata može se produljiti ovim recipročnim kretanjem, zato što su tako otporniji na ciklički zamor negoli na kontinuiranu rotaciju $(12,13)$. Frekvencija, apikalna snaga instrumenta, pritisak operatera te povećanje kanala, također su klinič- 
which also affect the resistance of files to cyclic fatigue (14).

Science advances, and single-use instrumentation systems have been created to prevent fracture from overuse. The files are used in a single tooth, in 1 to 4 root canals (as in molars) and are safe in narrow and curved canals (15). However, several studies have suggested that their traction and compression strengths are a disadvantage in the case of continuous rotation instruments (16).

In relation to the assessment of the separated fragment lengths, it is preferable that the longest fragment should remain inside the tooth to make its removal easier.

Therefore, this study was designed to test the null hypothesis that there were no significant differences between size 25 files F360 (Komet Dental, Lemgo, Germany), F6 SkyTaper (Komet Dental, Lemgo, Germany), Hyflex EDM (Coltene, Altstätten, Switzerland), iRace (FKG Dentaire, La Chaux-de-Fonds, Switzerland), Neoniti (Neolix, Chatres-LaForet, France), One Shape (Micro-Mega, Besançon, France) Protaper Next (Dentsply Maillefer, Ballaigues, Switzerland), Reciproc (VDW, Munich, Germany), Revo-S (Micro-Mega, Besançon, France) and Wave One Gold (Dentsply Maillefer, Ballaigues, Switzerland) in terms of resistance to cyclic fatigue and separated fragment lengths.

\section{Material and methods}

New size 25 files of the systems studied were selected ( $\mathrm{n}=30$ per system, total 300$)$. The systems were divided into groups in alphabetical order (Table 1).

The instruments were rotated using X-Smart Plus endo motor (Dentsply Maillefer, Ballaigues, Switzerland) (Fig. 1) at the speed and torque recommended by manufacturers. The speed and torque of each group using only the size 25 files of endodontic systems were: ki čimbenici koji utječu na otpornost instrumenta na ciklički zamor (14).

Kako bi se izbjegle frakture zbog istrošenosti, proizvedeni su sustavi s instrumentima za jednu instrumentaciju. Iglice se koriste u jednom zubu, u jednom do četiri korijenska kanala (npr. za kutnjake - molare) te su sigurne u uskim i zakrivljenim kanalima (15). No, u nekoliko istraživanja autori ističu da su otpornost na vlak i tlak nedostatak u slučaju kontinuirane rotacije (16).

U odnosu prema procjeni duljine separiranih fragmenata, poželjno je da najdulji fragment ostane u zubu kako bi se lakše mogao izvaditi.

Ovo istraživanje zamišljeno je tako da je nulta hipoteza bila da nema značajnih razlika u otpornosti na ciklički zamor te duljinu frakturiranih dijelova instrumenata između iglica veličine 25 sustava F360 (Komet Dental, Lemgo, Njemačka), F6 SkyTaper (Komet Dental, Lemgo, Njemačka), Hyflex EDM (Coltene, Altstätten, Švicarska), iRace (FKG Dentaire, La Chaux-de-Fonds, Švicarska), Neoniti (Neolix, ChartresLa-Foret, Francuska), One Shape (Micro-Mega, Besançon, Francuska), Protaper Next (Dentsply Maillefer, Ballaigues, Švicarska), Reciproc (VDW, München, Njemačka), Revo$S$ (Micro-Mega, Besançon, Francuska) te Wave One Gold (Dentsply Maillefer, Ballaigues, Švicarska).

\section{Materijali i metode}

Odabrane su nove iglice veličine 25 svih navedenih sustava ( $\mathrm{n}=30$ po sustavu, ukupno 300 iglica). Sustavi su poredani abecednim redom i podijeljeni u skupine (tablica 1.).

Instrumenti su pokretani endodontskim mikromotorom X-Smart Plus (Dentsply Maillefer, Ballaigues, Švicarska) (slika 1.) brzinom koju je preporučio proizvođač. Brzina i okretni moment svake skupine u kojoj su korištene samo iglice veličine 25 svih endodontskih sustava bili su:

Table 1 Systems and their characteristics.

Tablica 1. Sustavi i njihove karakteristike

\begin{tabular}{|c|c|c|c|c|}
\hline & System • Sustav & $\begin{array}{c}\text { Size/Taper } \bullet \\
\text { Veličina/konus }\end{array}$ & Alloy• Legura & Cross-section $\bullet$ Presjek \\
\hline $\begin{array}{l}\text { Group } 1 \\
\text { Skupina } 1\end{array}$ & F360 & $25 / 0.04$ & $\begin{array}{l}\text { conventional } \mathrm{NiTi} \\
\text { konvencionalni } \mathrm{Ni}-\mathrm{Ti} \\
\end{array}$ & \begin{tabular}{|l|} 
Double-S \\
dvostruko $S$ \\
\end{tabular} \\
\hline $\begin{array}{l}\text { Group } 2 \\
\text { Skupina } 2\end{array}$ & F6 SkyTaper & $25 / 0.06$ & $\begin{array}{l}\text { Conventional NiTi } \\
\text { konvencionalni } \mathrm{Ni}-\mathrm{Ti}\end{array}$ & $\begin{array}{l}\text { Double-S } \\
\text { dvostruko } S\end{array}$ \\
\hline $\begin{array}{l}\text { Group } 3 \\
\text { Skupina } 3\end{array}$ & Hyflex EDM & $25 /$ & CM-Wire & $\begin{array}{l}\text { Variable } \\
\text { Različiti }\end{array}$ \\
\hline $\begin{array}{l}\text { Group } 4 \\
\text { Skupina } 4\end{array}$ & iRace & $25 / 0.04$ & $\begin{array}{l}\text { Conventional NiTi } \\
\text { konvencionalni } \mathrm{Ni}-\mathrm{Ti}\end{array}$ & $\begin{array}{l}\text { Triangula } \\
\text { trokutasti }\end{array}$ \\
\hline $\begin{array}{l}\text { Group } 5 \\
\text { Skupina } 5\end{array}$ & Neoniti & $25 / 0.08$ & CM-Wire & $\begin{array}{l}\text { non-homothetic rectangular } \\
\text { pravokutni nehomotetički }\end{array}$ \\
\hline $\begin{array}{l}\text { Group } 6 \\
\text { Skupina } 6\end{array}$ & One Shape & $25 / 0.06$ & $\begin{array}{l}\text { conventional } \mathrm{NiTi} \\
\text { konvencionalni } \mathrm{Ni}-\mathrm{Ti}\end{array}$ & $\begin{array}{l}\text { variable } \\
\text { različiti }\end{array}$ \\
\hline $\begin{array}{l}\text { Group } 8 \\
\text { Skupina } 8\end{array}$ & Reciproc & $25 / 0.08$ & M-Wire & $\begin{array}{l}\text { S-shaped } \\
\text { oblik slova } S\end{array}$ \\
\hline $\begin{array}{l}\text { Group } 9 \\
\text { Skupina } 9\end{array}$ & Revo-S & $25 / 0.06$ & $\begin{array}{l}\text { conventional } \mathrm{NiTi} \\
\text { konvencionalni } \mathrm{Ni}-\mathrm{Ti}\end{array}$ & $\begin{array}{l}\text { asymmetrical } \\
\text { asimetrični }\end{array}$ \\
\hline $\begin{array}{l}\text { Group } 10 \\
\text { Skupina } 10\end{array}$ & Wave One Gold & $25 / 0.07$ & Gold-Wire & $\begin{array}{l}\text { rectangular } \\
\text { pravokutni }\end{array}$ \\
\hline
\end{tabular}


Group 1 (F360): $300 \mathrm{rpm}, 1.8$ N.cm and continuous rotation.

Group 2 (F6 SkyTaper): 300 rpm, 2.2 N.cm and continuous rotation.

Group 3 (Hyflex EDM): 500 rpm, 2.5 N.cm and continuous rotation.

Group 4 (iRace): 600 rpm, 1.5 N.cm and continuous rotation.

Group 5 (Neoniti): $400 \mathrm{rpm}, 1.5 \mathrm{~N} \cdot \mathrm{cm}$ and continuous rotation.

Group 6 (One Shape): $400 \mathrm{rpm}, 4 \mathrm{~N} \cdot \mathrm{cm}$ and continuous rotation.

Group 7 (Protaper Next): $300 \mathrm{rpm}, 2 \mathrm{~N} \cdot \mathrm{cm}$ and continuous rotation.

Group 8 (Reciproc): $300 \mathrm{rpm}, 2 \mathrm{~N} \cdot \mathrm{cm}$ and reciprocation motion.

Group 9 (Revo-S): 350 rpm, 0.8 N.cm and continuous rotation.

Group 10 (Wave One Gold): 350 rpm, $2 \mathrm{~N} \cdot \mathrm{cm}$ and reciprocation motion.

The instruments were firmly held with clamping mechanism (Fig. 2) with passive adjustment and without pressure in a stainless-steel block containing an artificial canal with the following characteristics: $60^{\circ}$ curvature, radius of curvature $3.5 \mathrm{~mm}$, length $21 \mathrm{~mm}$, width $2 \mathrm{~mm}$, and depth $3 \mathrm{~mm}$. The characteristics of model were similar to the block used by Gambarini et al. (17) and Champa et al. (18). The canal was lubricated with glycerin after each file.

The time was calculated in seconds (s) until fracture. The number of cycles to fracture (NCF) was calculated by the following formula: (Resistance (s) x Speed)/60. The separated fragment lengths were measured with a digital Vernier caliper (Fig. 3).

Statistical analysis was performed with the SPSS 18 programme at a 95\% confidence level, using the Levene's Test skupina 1 (F360): $300 \mathrm{rpm}, 1,8 \mathrm{~N}$ cm i kontinuirana rotacija; skupina 2 (F6 SkyTaper): 300 rpm, 2,2 N cm i kontinuirana rotacija;

skupina 3 (Hyflex EDM): 500 rpm, 2,5 N cm i kontinuirana rotacija;

skupina 4 (iRace): 600 rpm, 1,5 N cm i kontinuirana rotacija; skupina 5 (Neoniti): $400 \mathrm{rpm}, 1,5 \mathrm{~N}$ cm i kontinuirana rotacija;

skupina 6 (One Shape): $400 \mathrm{rpm}, 4 \mathrm{~N}$ cm i kontinuirana rotacija;

skupina 7 (Protaper Next): 300 rpm, $2 \mathrm{~N} \mathrm{~cm} \mathrm{i} \mathrm{kontinuira-}$ na rotacija;

skupina 8 (Reciproc): $300 \mathrm{rpm}, 2 \mathrm{~N}$ cm i recipročni pokret; skupina 9 (Revo-S): $350 \mathrm{rpm}, 0,8 \mathrm{~N} \mathrm{~cm}$ i kontinuirana rotacija;

skupina 10 (Wave One Gold): 350 rpm, 2 N cm i recipročni pokret.

Instrumente je čvrsto držao škripac (slika 2.) s pasivnom prilagodbom i bez pritiska, na čeličnom bloku koji je imao umjetno napravljeni kanal sa sljedećim karakteristikama: zavoj od $60^{\circ}$, radijus zavoja $3,5 \mathrm{~mm}$, duljina $21 \mathrm{~mm}$, širina 2 $\mathrm{mm}$ i dubina $3 \mathrm{~mm}$. Karakteristike modela bile su slične blokovima kojima su se koristili Gambarini i suradnici (17) te Champa i njegovi kolege (18). Kanal je vlažen glicerinom nakon svake iglice.

Vrijeme do puknuća mjereno je u sekundama (s). Broj ciklusa potreban da se dogodi puknuće (NCF) izračunat je sljedećom formulom: otpor $(\mathrm{s}) \times$ brzina $/ 60$. Separirani, frakturirani fragmenti mjereni su Vernierovom digitalnom pomičnom mjerkom (slika 3.).

Statistička analiza obavljena je u programu SPSS 18 na 95-postotnom intervalu pouzdanosti s pomoću Levenova testa za usporedbu varijanci, Welchova testa za usporedbu srednjih vrijednosti te Games-Howellova testa za otkrivanje razlika između skupina.
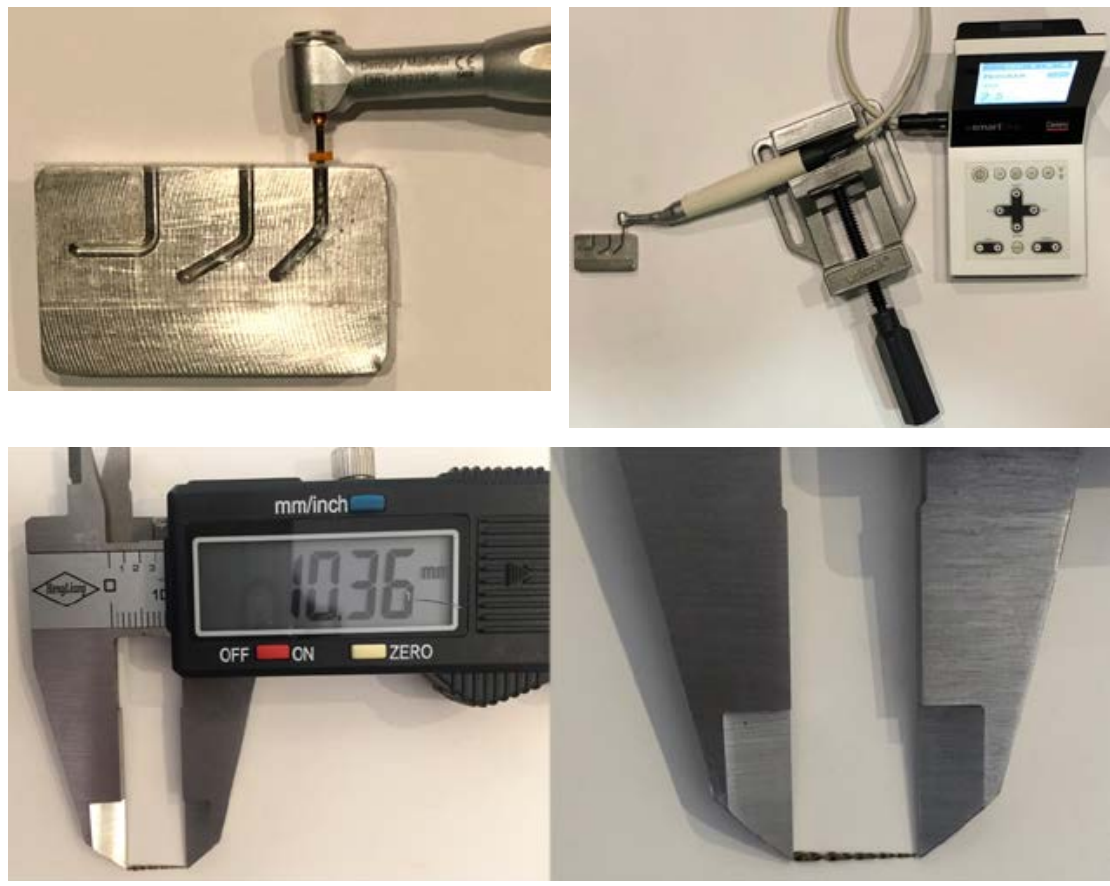

Figure 1. Hyflex EDM 25/ file in an artificial $60^{\circ}$ canal.

Slika 1. Instrument Hyflex EDM veličine $25 \mathrm{u}$ umjetnom kanalu sa zavojem od $60^{\circ}$

Figure 2. Clamping mechanism of the handpiece for the X-Smart Plus endo motor.

Slika 2. Škripac s količnikom endodontskog mikromotora X-Smart Plus

Figure 3. Measurement of separated fragment of Wave One Gold Primary (25/0.07) file with digital Vernier caliper.

Slika 3. Mjerenje separiranog fragmenta instrumenta sustava Wave One Gold Primary $(25 / 0,07)$ s pomoću Vernierove digitalne pomične mjerke 
to compare variances, the Welch's Test to compare means, and the Games-Howell's Test to reveal differences between groups.

\section{Results}

In all of the comparisons that were made, the Levene's Test was carried out, and no equal variances were assumed $(\mathrm{P}<0.05)$; therefore, it was decided to carry out the Welch's Test (Tables 2, 3 and 4).

The cyclic fatigue mean values and statistics are presented in Table 2. Statistically, in terms of resistance, Neoniti and Hyflex EDM were superior to other systems $(\mathrm{P}<0.05)$, but there were no significant differences between Wave One

\section{Rezultati}

U svim je usporedbama obavljen Levenov test te, kako nigdje nisu pronađene iste varijance $(\mathrm{P}<0,05)$, proveden je Welchov test (tablice 2, 3 i 4). Srednje vrijednosti cikličkog zamora i statistički podatci nalaze se u tablici 2. Statistički, kad je riječ o otporu, sustavi Neoniti i Hyfelx EDM bili su bolji od ostalih $(\mathrm{P}<0,05)$, ali nije bilo statistički značajnih razlika između sustava Wave One Golda i F6 SkyTaper $(\mathrm{P}=$ $1,000)$, sustava One Shape i Protaper Next $(\mathrm{P}=0,121)$, su-

Table 2. Means and statistics for resistance to cyclic fatigue (s).

Tablica 2. Srednje vrijednosti i statistički podatci o otpornosti na ciklički zamor (s)

\begin{tabular}{c|c|c|c|c|c|c|c|c|c|c|}
\hline F360 & F6 & Hyflex & iRace & Neoniti & O.Shape & P.Next & Reciproc & R-S & WOG \\
\hline $\begin{array}{c}152.43 \pm \\
11.25\end{array}$ & $190.83 \pm$ & $\underline{331.07 \pm}$ & $27.37 \pm$ & $\underline{414.83 \pm}$ & $56.23 \pm$ & $70.43 \pm$ & $168.67 \pm$ & $33.53 \pm$ & $188.00 \pm$ \\
\hline
\end{tabular}
0.000

Welch's Test $\bullet$ Welchov test

0.000

\begin{tabular}{|c|c|c|c|c|c|c|c|c|c|c|c|c|c|c|}
\hline \multicolumn{10}{c}{ Games-Howell's Test • Games-Howellov test } \\
\hline & F360 & F6 & Hyflex & iRace & Neoniti & O.Shape & P.Next & Reciproc & R-S & WOG \\
\hline F360 & - & 0.009 & 0.000 & 0.000 & 0.000 & 0.000 & 0.000 & 0.765 & 0.000 & 0.001 \\
\hline F6 & 0.009 & - & 0.000 & 0.000 & 0.000 & 0.000 & 0.000 & 0.599 & 0.000 & 1.000 \\
\hline Hyflex & 0.000 & 0.000 & - & 0.000 & 0.001 & 0.000 & 0.000 & 0.000 & 0.000 & 0.000 \\
\hline iRace & 0.000 & 0.000 & 0.000 & - & 0.000 & 0.000 & 0.000 & 0.000 & 0.550 & 0.000 \\
\hline Neoniti & 0.000 & 0.000 & 0.001 & 0.000 & - & 0.000 & 0.000 & 0.000 & 0.000 & 0.000 \\
\hline O.Shape & 0.000 & 0.000 & 0.000 & 0.000 & 0.000 & - & 0.121 & 0.000 & 0.001 & 0.000 \\
\hline P.Next & 0.000 & 0.000 & 0.000 & 0.000 & 0.000 & 0.121 & - & 0.000 & 0.000 & 0.000 \\
\hline Reciproc & 0.765 & 0.599 & 0.000 & 0.000 & 0.000 & 0.000 & 0.000 & - & 0.000 & 0.556 \\
\hline R-S & 0.000 & 0.000 & 0.000 & 0.550 & 0.000 & 0.001 & 0.000 & 0.000 & - & 0.000 \\
\hline WOG & 0.001 & 1.000 & 0.000 & 0.000 & 0.000 & 0.000 & 0.000 & 0.556 & 0.000 & - \\
\hline
\end{tabular}

Table 3. Means and statistics of number of cycles to fracture (NCF).

Tablica 3. Srednje vrijednosti i statistički podatci o broju ciklusa do frakture (NCF)

\begin{tabular}{|c|c|c|c|c|c|c|c|c|c|c|}
\hline F360 & F6 & Hyflex & iRace & Neoniti & O.Shape & P.Next & Reciproc & R-S & WOG \\
\hline $762.17 \pm$ & 954.16 & $\underline{2758.88}$ & 273.67 & $\underline{2765.55}$ & 374.88 & 352.16 & 843.33 & 195.61 & 1096.66 \\
56.25 & \pm 83.06 & \pm 210.14 & \pm 26.54 & \pm 171.07 & \pm 55.90 & \pm 25.97 & \pm 76.68 & \pm 31.77 & \pm 66.42 \\
\hline
\end{tabular}

Levene's Test $\bullet$ Leveneov test

0,000

Welch's Test • Welchov test

0,000

\begin{tabular}{|c|c|c|c|c|c|c|c|c|c|c|}
\hline \multicolumn{11}{|c|}{ Games-Howell's Test • Games-Howellov test } \\
\hline & $\mathrm{F} 360$ & F6 & Hyflex & iRace & Neoniti & O.Shape & P.Next & Reciproc & R-S & WOG \\
\hline F360 & - & 0.009 & 0.000 & 0.000 & 0.000 & 0.000 & 0.000 & 0.765 & 0.000 & 0.000 \\
\hline F6 & 0.009 & - & 0.000 & 0.000 & 0.000 & 0.000 & 0.000 & 0.599 & 0.000 & 0.183 \\
\hline Hyflex & 0.000 & 0.000 & - & 0.000 & 1.000 & 0.000 & 0.000 & 0.000 & 0.000 & 0.000 \\
\hline iRace & 0.000 & 0.000 & 0.000 & - & 0.000 & 0.049 & 0.002 & 0.000 & 0.010 & 0.000 \\
\hline Neoniti & 0.000 & 0.000 & 1.000 & 0.000 & - & 0.000 & 0.000 & 0.000 & 0.000 & 0.000 \\
\hline O.Shape & 0.000 & 0.000 & 0.000 & 0.049 & 0.000 & - & 0.999 & 0.000 & 0.000 & 0.000 \\
\hline P.Next & 0.000 & 0.000 & 0.000 & 0.002 & 0.000 & 0.999 & - & 0.000 & 0.000 & 0.000 \\
\hline Reciproc & 0.765 & 0.599 & 0.000 & 0.000 & 0.000 & 0.000 & 0.000 & - & 0.000 & 0.000 \\
\hline R-S & 0.000 & 0.000 & 0.000 & 0.010 & 0.000 & 0.000 & 0.000 & 0.000 & - & 0.000 \\
\hline WOG & 0.000 & 0.183 & 0.000 & 0.000 & 0.000 & 0.000 & 0.000 & 0.000 & 0.000 & - \\
\hline
\end{tabular}


Table 4. Means and statistics of separated fragments length $(\mathrm{mm})$.

Tablica 4. Srednje vrijednosti i statistički podatci o duljinama separiranih fragmenata $(\mathrm{mm})$

\begin{tabular}{|c|c|c|c|c|c|c|c|c|c|c|}
\hline & F360 & F6 & Hyflex & iRace & Neoniti & O.Shape & P.Next & Reciproc & R-S & WOG \\
\hline & $\frac{10.48 \pm}{\underline{0.19}}$ & $9.45 \pm 0.34$ & $9.86 \pm 0.42$ & $8.06 \pm 0.07$ & $\begin{array}{c}10.27 \pm \\
0.30\end{array}$ & $9.09 \pm 0.12$ & $9.90 \pm 0.39$ & $\frac{11.44 \pm}{\underline{0.21}}$ & $\begin{array}{c}10.05 \pm \\
0.55\end{array}$ & $9.85 \pm 0.39$ \\
\hline
\end{tabular}

Levene's Test $\bullet$ Leveneov test

0,000

Welch's Test • Welchov test

0,000

\begin{tabular}{|c|c|c|c|c|c|c|c|c|c|c|c|c|}
\hline \multicolumn{10}{c|}{ Games-Howell's Test • Games-Howellov test } \\
\hline & F360 & F6 & Hyflex & iRace & Neoniti & O.Shape & P.Next & Reciproc & R-S & WOG \\
\hline F360 & - & 0.000 & 0.183 & 0.000 & 0.968 & 0.000 & 0.191 & 0.000 & 0.880 & 0.103 \\
\hline F6 & 0.000 & - & 0.853 & 0.000 & 0.017 & 0.596 & 0.745 & 0.000 & 0.669 & 0.849 \\
\hline Hyflex & 0.183 & 0.853 & - & 0.000 & 0.834 & 0.027 & 1.000 & 0.000 & 1.000 & 1.000 \\
\hline iRace & 0.000 & 0.000 & 0.000 & - & 0.000 & 0.000 & 0.000 & 0.000 & 0.000 & 0.000 \\
\hline Neoniti & 0.968 & 0.017 & 0.834 & 0.000 & - & 0.000 & 0.874 & 0.000 & 0.999 & 0.752 \\
\hline O.Shape & 0.000 & 0.596 & 0.027 & 0.000 & 0.000 & - & 0.010 & 0.000 & 0.042 & 0.016 \\
\hline P.Next & 0.191 & 0.745 & 1.000 & 0.000 & 0.874 & 0.010 & - & 0.000 & 1.000 & 1.000 \\
\hline Reciproc & 0.000 & 0.000 & 0.000 & 0.000 & 0.000 & 0.000 & 0.000 & - & 0.001 & 0.000 \\
\hline R-S & 0.880 & 0.669 & 1.000 & 0.000 & 0.999 & 0.042 & 1.000 & 0.001 & - & 1.000 \\
\hline WOG & 0.103 & 0.849 & 1.000 & 0.000 & 0.752 & 0.016 & 1.000 & 0.000 & 1.000 & - \\
\hline
\end{tabular}

Gold vs F6 SkyTaper $(\mathrm{P}=1.000)$, One Shape vs Protaper Next ( $\mathrm{P}=0.121)$, Reciproc vs F360 ( $\mathrm{P}=0.765)$, Reciproc vs Wave One Gold ( $\mathrm{P}=0.556)$ and Revo-S vs iRace $(\mathrm{P}=0.550)$.

The NCF were described in Table 3. Neoniti and Hyflex EDM proved to be statistically superior to other systems $(\mathrm{P}<0.000)$. However, there were no significant differences between One Shape vs Protaper Next $(\mathrm{P}=0.999)$, Wave One Gold vs F6 SkyTaper $(\mathrm{P}=0.183)$, Neoniti vs Hyflex EDM ( $\mathrm{P}=1.000)$, Reciproc vs F360 ( $\mathrm{P}=0.765)$ and Reciproc vs F6 SkyTaper $(\mathrm{P}=0.599)$.

The separated fragment length mean values and statistics are presented in Table 4. The highest values were obtained by F360 and Reciproc. F360 was significantly superior $(\mathrm{P}=0.000)$ vs F6 SkyTaper, One Shape and iRace. Reciproc was significantly superior to all the other systems $(p \leq 0.001)$.

\section{Discussion}

In the present study, files with continuous and reciprocating motion and with different alloys, cross-sections, tapers, speeds and torques were studied. The results showed that the systems with CM-Wire and Gold-Wire alloys, reciprocating motion and conventional NiTi instruments with an $\mathrm{S}$ crosssection offered better resistance to cyclic fatigue.

A complete bio-mechanical preparation of root canals is an essential factor for endodontic success. Shaping and cleaning of the canal are performed during this phase, and they present great difficulty in curved canals (19-21). Goldberg et al. (22) reported that apical enlargement may produce defects such as apical transportation or zipping, with a risk of endodontic treatment failure.

The cross-section design, the chemical composition of the alloy and the thermo-mechanical process used during the manufacture of the alloy all influence cyclic fatigue (23-25). In the present study, F6 SkyTaper, with a S-shaped cross-section, obtained a higher NCF than other conventional Ni- stava Reciproc i F360 (P = 0,765), sustava Reciproc i Wave One Gold $(\mathrm{P}=0,556)$ te sustava Revo-S i iRace $(\mathrm{P}=0,550)$.

Vrijednosti NCF-a prikazane su tablici 3. Neoniti i Hyflex EDM bili su statistički bolji od ostalih sustava $(\mathrm{P}<0,000)$. No, nije bilo statistički značajnih razlika između sustava One Shape i Protaper Next $(\mathrm{P}=0,999)$, sustava Wave One Gold i F6 SkyTaper $(\mathrm{P}=0,183)$, sustava Neoniti i Hyflex EDM (P $=1,000)$, sustava Reciproc i F360 $(\mathrm{P}=0,765)$ te Reciproca $\mathrm{i}$ F6 SkyTapera $(\mathrm{P}=0,599)$.

Srednje vrijednosti duljina separiranih fragmenata i statistički podatci nalaze se u tablici 4. Najveće vrijednosti imali su sustavi F360 i Reciproc. Sustav F360 bio je značajno bolji $(\mathrm{P}=0,000)$ od sustava F6 SkyTaper, One Shape i iRace. Sustav Reciproc bio je značajno bolji od svih drugih ( $\mathrm{p} \leq 0,001)$.

\section{Rasprava}

U ovom istraživanju ispitivane su iglice $s$ kontinuiranim i recipročnim kretanjem izrađene od različitih legura, $s$ različitim presjecima, konusima, brzinama i okretnim momentima. Rezultati su pokazali da su sustavi izrađeni od legura CM-wire i Gold-Wire, s recipročnim kretanjem, te konvencionalni $\mathrm{Ni}$-Ti instrumenti s presjekom u obliku slova $S$ otporniji na ciklički zamor.

Kompletna biomehanička preparacija korijenskih kanala ključni je čimbenik za uspjeh endodontskog liječenja. Oblikovanje i čišćenje kanala obavlja se tijekom te faze terapije, a u slučaju zakrivljenih kanala to je posebno otežavajuće (19 21). Goldberg i suradnici (22) naveli su da apikalno povećanje može uzrokovati defekte kao što su prijenos preko apeksa, ili tzv. zipping (eliptično proširenje apeksa) koji mogu završiti neuspjehom.

Presjek, kemijski sastav legure i termomehanički proces korišten tijekom izrade legure utječu na ciklički zamor (23 25). U ovom istraživanju uočeno je da je F6 SkyTaper, koji 
Ti systems such as iRace, One Shape and Revo-S, indicating that an S-shaped cross-section offered more flexibility and resistance. On the other hand, CM-Wire alloy (Neoniti and Hyflex EDM) obtained the best results compared to the other systems studied, showing that CM-Wire with memory control is more resistant than the other alloys.

The cyclic fatigue findings of this study (Tables 2 and 3 ) showed that Group 4 (iRace, 27.37 \pm 2.65 s, 273.67 \pm 26.54 NCF) and Group 9 (Revo-S, 33.53 \pm 5.45 s, 195.61 \pm 31.77 $\mathrm{NCF}$ ) obtained worse results than the other groups. In contrast, Group 3 (Hyflex EDM, 331.07 $\pm 25.22 \mathrm{~s}$, $2758.88 \pm 210.14$ NCF) and Group 5 (Neoniti, $414.83 \pm 25.66 s, 2765.55 \pm 171.07 \mathrm{NCF}$ ) achieved significantly better results than the other systems. It may also be observed that the S-shaped cross-sectional systems (F360, F6 SkyTaper and Reciproc) obtained better results than the conventional NiTi and M-Wire systems (iRace, Protaper Next, One Shape and Revo-S). Reciprocating motion (Reciproc and Wave One Gold) was found to improve the cyclic fatigue results compared to almost all the continuous motion systems studied (F360, iRace, Protaper Next, One Shape and Revo-S). Regarding separated fragment lengths, F360 $(10.48 \pm 0.19 \mathrm{~mm})$ and Reciproc $(11.44 \pm 0.21 \mathrm{~mm})$ obtained the highest values, while iRace $(8.06 \pm 0.07 \mathrm{~mm})$ and One Shape $(9.09 \pm 0.12 \mathrm{~mm})$ presented the lowest fragments length.

Aminsobhani et al. (26) compared the cyclic fatigue and separated fragment lengths of size 25 Neoniti, Race, Mtwo, $\mathrm{TF}$ and Protaper Next files with continuous rotation in 3 simulated canals in a stainless-steel block. In all 3 canals, the Neoniti system obtained better results than other systems: between 400 and 1600 NCF. Statistically, Neoniti was superior to other systems, concluding that CM-Wire (Neoniti) is better than conventional NiTi, R-Phase and M-Wire. For separated fragment lengths, the Race system obtained the lowest mean and TF the highest in group 1. In group 2, Neoniti had the lowest average whereas Race had the highest; and in group 3, Neoniti obtained the lowest mean and TF the highest. In the present study, unlike Aminsobhani et al., Neoniti obtained better fatigue results with continuous rotation also, but both studies agree that Neoniti (CM-Wire) was more resistant than Race with conventional NiTi. As regards separated fragment lengths, the present study is congruent with Aminsobhani et al.'s findings for Group 1, where Race obtained a lower average than Neoniti, unlike in Groups 2 and 3, probably because of the characteristics of each artificial canal in this case.

Kaval et al. (27) investigated the cyclic fatigue of F6 SkyTaper, One Shape, K3XF and TRUShape 3D (Dentsply Tulsa Dental Specialties, Tulsa, USA) files using model with similar characteristics of the block of this study; they observed significant differences between all groups, with F6 SkyTaper obtaining the highest resistance $(959 \pm 92 \mathrm{NCF})$. Similar to the findings in the present study, Kaval et al. considered that a double-S cross-section could improve the resistance to $\mathrm{cy}$ clic fatigue. Furthermore, F6 SkyTaper was significantly superior to One Shape, as found by Kaval et al.

Pedullà et al. (28) compared size 25 Hyflex EDM, Reciproc and Wave One files in an artificial canal with $60^{\circ} \mathrm{cur}-$ ima presjek u obliku slova $S$, imao viši NCF od drugih konvencionalnih Ni-Ti sustava kao što su iRace, One Shape i Revo-S, što upućuje na to da takav presjek instrumenta omogućuje veću fleksibilnost i bolji otpor. $S$ druge strane, legurom CM-Wire (Neoniti i Hyflex EDM) postignuti su najbolji rezultati u usporedbi $s$ drugim ispitivanim sustavima, što pokazuje da je CM-Wire s memorijskom kontrolom otporniji od drugih legura. Nalazi cikličkog zamora u ovom istraživanju (tablice 2. i 3.) pokazali su da su skupina 4 (iRace, NCF vrijednosti 27,37 $\pm 2,65 s, 273,67 \pm 26,54)$ i skupina 9 (Revo-S, NCF vrijednosti $33,53 \pm 5,45 \mathrm{~s}, 195,61 \pm 31,77$ ) imale lošije rezultate od drugih ispitivanih sustava. Suprotno tomu, skupina 3 (Hyflex EDM, vrijednosti NCF-a 331,07 $\pm 25,22$ s, $2758,88 \pm 210,14$ ) i skupina 5 (Neoniti, vrijednosti NCF-a $414,83 \pm 25,66 \mathrm{~s}, 2765,55 \pm 171,07)$ imale su značajno bolje rezultate od svih preostalih sustava. Moglo se također uočiti da su sustavi koji imaju presjek instrumenta u obliku slova $S$ (F360, F6 SkyTaper i Reciproc) imali bolje rezultate od konvencionalnih sustava Ni-Ti i M-Wire (iRace, Protaper Next, One Shape i Revo-S). Recipročni pokret (Reciproc i Wave One Gold) pokazao se boljim kad je riječ o cikličkom zamoru u odnosu prema gotovo svim ostalim sustavima s kontinuiranim pokretom (F360, iRace, Protaper Next, One Shape i Revo-S). Kad je riječ o duljini frakturiranih segmenata, sustavi F360 $(10,48 \pm 0,19 \mathrm{~mm})$ i Reciproc $(11,44 \pm 0,21 \mathrm{~mm})$ imali su najveće vrijednosti, a iRace $(8,06 \pm 0,07 \mathrm{~mm})$ i One Shape $(9,09 \pm 0,12 \mathrm{~mm})$ najkraće duljine frakturiranih segmenata.

Aminsobhani i suradnici (26) uspoređivali su ciklički zamor i duljine separiranih fragmenata instrumenata veličine 25 sustava Neoniti, Race, Mtwo, TF i Protaper Next s kontinuiranim pokretom okretanja na trima simulacijama kanala u čeličnim blokovima. U svima je Neoniti imao bolje rezultate od ostalih sustava - između 400 i 1600 NCF-a. Statistički je Neoniti imao bolje rezultate od svih ostalih sustava, na temelju čega su zaključili da je CM-Wire bolja legura od konvencionalnih legura Ni-Ti, R-Phase i M-Wire. Kad je riječ o frakturiranim segmentima, sustav Race imao je najmanju srednju vrijednost, a TF najveću u skupini 1 . U skupini 2, Neoniti je imao najmanju srednju vrijednost, a Race najveću, a u skupini 3 Neoniti je imao bolje rezultate zamora pri kontinuiranoj rotaciji. Oba istraživanja zaključena su tvrdnjom da je Neoniti (CM-Wire) otporniji od sustava Race izrađenog od konvencionalne nikal-titanijeve legure. Istaknimo da je u vezi s duljinom separiranih fragmenata, naše istraživanje u skladu s istraživanjem Aminsobhanija i suradnika kad je riječ o skupini 1, u kojoj je sustav Race imao nižu srednju vrijednost od sustava Neoniti, za razliku od skupina 2 i 3, što se može pripisati karakteristikama umjetnih kanala na kojima su sustavi ispitivani.

Kaval i suradnici (2) istraživali su ciklički zamor sustava F6 SkyTaper, One Shape, K3XF i TRUShape 3D (Dentsply Tulsa Dental Specialties, Tulsa, SAD) na modelu sličnom našemu. Uočili su značajne razlike između svih skupina, s tim da je F6 SkyTaper imao najbolje vrijednosti otpornosti (NCF $959 \pm 92$ ). Slično našim nalazima, Kaval i suradnici smatraju da presjek u obliku dvostrukoga slova $S$ može poboljšati otpor prema cikličkom zamoru. Nadalje, prema tom istraživanju, sustav F6 SkyTaper bio je značajno bolji od One Shapea. 
vature with a $3 \mathrm{~mm}$ radius. Hyflex EDM gave better results than Reciproc and Wave One, but no significant differences were observed between the latter two. The authors concluded that Hyflex EDM was more resistant than other systems, determining that CM-Wire was more resistant than M-Wire. In relation to movement, the reciprocating motion did not affect the results. Like Pedullà et al., the Hyflex EDM results were statistically superior to Reciproc in a canal with the same curvature in the present study. Unlike Pedullà et al., in this investigation the reciprocating motion improved the results of other files except in instruments with CM-Wire.

Ersoy et al. (29) studied F360, TF, FlexMaster and Race in a stainless-steel block with an artificial canal measuring $1.5 \mathrm{~mm}$ in diameter with a $60^{\circ}$ curvature. They observed that F360 was significantly more resistant than the other systems; TF was significantly more resistant than FlexMaster and Race, and there were no significant differences between FlexMaster and Race. They concluded that F360 was the best system, showing that the S-shaped cross-section in systems with continuous rotation improved the cyclic fatigue resistance between systems with shape memory; furthermore, TF was shown to be superior to FlexMaster and Race. The results of the present study, which also compared the files in a $60^{\circ}$ canal, are similar to the results obtained by Ersoy et al. in that F360 was significantly better than Race.

Topçuoğlu et al. (30) examined size 25 Wave One Gold, Reciproc and Wave One files in an artificial double $S$ canal, $1.4 \mathrm{~mm}$ in diameter and $18 \mathrm{~mm}$ in length. The results showed that Wave One Gold obtained the best results in apical and coronal curvature. In the statistical analysis, Wave One Gold was significantly better than Reciproc and Wave One in both curvatures, whereas Reciproc was superior to Wave One in the apical curve; however, there were no significant differences between the latter two systems in the coronal curvature. In conclusion, the authors found that Wave One Gold offered the best resistance in an artificial double $S$ canal. In the present study, Wave One Gold was superior to Reciproc, with similar results to those of Topçuoğlu et al, but there were significant differences in number of cycles to fracture, probably due to the speed and alloy of Wave One Gold.

A study by Keskin et al. (31) compared the resistance to cyclic fatigue and the separated fragments length of size 25 Reciproc Blue, Reciproc and Wave One Gold files at $60^{\circ}$ curvature with different radius of curvature. The authors determined that Reciproc Blue obtained the highest significant resistance to cyclic fatigue, and Wave One Gold was significantly better than Reciproc as found by Topçuoğlu et al, confirming that Gold-Wire improved the characteristics of $\mathrm{M}$ Wire. Regarding the length of the separated fragments, they observed no significant differences $(\mathrm{P}>0.05)$. In contrast to Keskin et al., the fracture times in the present study were similar for Wave One Gold and Reciproc except in NCF, and significant differences in separated fragment lengths were observed.

Gündogar et al. (32) examined size \#25 One Shape, Hyflex EDM, Wave One Gold and Reciproc Blue files at $60^{\circ}$ curvature and a $5 \mathrm{~mm}$ curvature radius. Hyflex EDM obtained significantly better resistance and One Shape was sig-
Pedullà i suradnici (28) uspoređivali su instrumente veličine 25 sustava Hyflex EDM, Reciproc i Wave One u umjetno stvorenim kanalima sa zavojem od $60^{\circ}$ i radijusom od 3 $\mathrm{mm}$. Hyflex EDM imao je bolje rezultate od sustava Reciproc i Wave One, ali nije bilo značajne razlike između tih dvaju sustava. Autori su zaključili da je Hyflex EDM otporniji od drugih, odnosno da je legura CM-Wire otpornija od M-Wirea. Kad je riječ o kretanju, recipročno pokretanje nije utjecalo na rezultate. Kao i u tom istraživanju, i u našemu su rezultati sustava Hyflex EDM bili bolji od onih za sustav Reciproc u kanalu s istim zakrivljenjem, no recipročno kretanje u našem je istraživanju poboljšalo rezultate svih drugih instrumenata izrađenih od legure CM-Wire.

Ersoy i suradnici (29) ispitivali su sustave F360, Tf, FlexMaster i Race u čeličnim blokovima s umjetnim kanalom promjera $1,5 \mathrm{~mm}$ i zakrivljenošću od $60^{\circ}$. Opazili su da je F360 značajno otporniji od ostalih sustava. TF je bio značajno otporniji od sustava FlexMaster i Race, a između njih nije bilo većih razlika. Zaključili su da je F360 najbolji sustav, potvrđujući da presjek u obliku slova $S$ u sustavima s kontinuiranom rotacijom poboljšava otpor prema cikličkom zamoru ako pamte oblik. Nadalje, TF se pokazao boljim od sustava FlexMaster i Race. Rezultati našeg istraživanja, u kojem se također uspoređivalo instrumente u kanalu sa zakrivljenošću od $60^{\circ}$, slični su rezultatima Ersoyja i suradnika jer je i kod njih F360 imao bolje rezultate od sustava Race.

Topçuoğlu i suradnici (30) ispitivali su instrumente veličine 25 sustava Wave One Gold, Reciproc i Wave One u umjetno napravljenom kanalu oblika dvostrukoga slova $S$ promjera $1,4 \mathrm{~mm}$ i duljine $18 \mathrm{~mm}$. Rezultati su pokazali da je sustav Wave One Gold najbolji u apikalnom i koronalnom zavoju. U statističkoj analizi je Wave One Gold bio značajno bolji od sustava Reciproc i Wave One u oba zavoja. Reciproc je bio bolji od Wave One u apikalnom zavoju, ali nije bilo značajnih razlika u koronalnom zavoju. U zaključcima su autori ustvrdili da Wave One Gold najotporniji u umjetnom kanalu u obliku dvostrukoga slova $S$. Wave One Gold bio je bolji od sustava Reciproc i u našem istraživanju - rezultati su bili sličnima onima u istraživanju Topçuoğlua i suradnika, ali je razlika bila značajna u broju ciklusa koji su uzrokovali frakturu, vjerojatno zbog brzine i legure sustava Wave One Gold.

Istraživanje Keskina i suradnika (31) uspoređivalo je otpor na ciklički zamor i duljinu frakturiranih segmenata instrumenata veličine 25 sustava Reciproc Blue, Reciproc i Wave One Gold u zavojima od $60^{\circ} \mathrm{s}$ različitim radijusom zakrivljenosti. Autori su ustvrdili da je sustav Reciproc Blue najotporniji na ciklički zamor, a Wave One Gold bio je značajno bolji od sustava Reciproc, slično kao i u spomenutom istraživanju Topçuoğlua i suradnika, što potvrđuje da legura Gold-Wire ima poboljšane karakteristike legure M-Wire. Kad je riječ o duljini frakturiranih fragmenata, nije bilo statistički značajnih razlika $(P>0,05)$. Suprotno tom istraživanju, $\mathrm{u}$ našem su vremena frakturiranja bila slična za sustave Wave One Gold i Reciproc, osim za NCF, a opažene su i znatne razlike u duljini separiranih fragmenata.

Gündogar i suradnici (32) također su ispitivali instrumente veličine 25 sustava One Shape, Hyflex EDM, Wave One Gold i Reciproc Blue u kanalima sa zavojem od $60^{\circ}$ i ra- 
nificantly the worst system; they found that the new alloys were better than the conventional $\mathrm{NiTi}$. As regards separated fragment lengths, they found no significant differences. They concluded that Hyflex EDM showed the highest resistance to cyclic fatigue fracture. The results of the study by Gündogar et al., are similar to those obtained in the present study: the Hyflex EDM was better than Wave One Gold and One Shape, determining that CM-Wire alloy with memory control improved the results of Gold-Wire and conventional NiTi. However, significant differences were observed in separated fragment lengths.

\section{Conclusions}

The systems with CM-Wire (Hyflex EDM and Neoniti) were superior to the other systems for cyclic fatigue. For separated fragment lengths, F360 (conventional NiTi) and Reciproc (M-Wire), the lengths were longer.

\section{Acknowledgements}

The authors thank Komet Dental, Endovations-FKG Dentaire, Coltene, Dentsply Maillefer, Neolix, VDW and Micro-Mega for providing the instruments.

\section{Conflict of interests}

The authors declare that there is no conflict of interest. dijusom od 5 mm. Hyflex EDM postigao je značajno bolju otpornost, a One Shape je bio najlošiji sustav. Ustvrdili su da su nove legure bolje od konvencionalne legure Ni-Ti. Kad je riječ o duljini separiranih fragmenata, nije bilo značajnih razlika. Zaključili su da je Hyflex EDM najotporniji na frakture zbog cikličkog zamora. Kao i u istraživanju Gündogara i suradnika, i u našem istraživanju otkriveno je da je Hyflex EDM bolji od sustava Wave One Gold i One Shape, što potvrđuje da je legura CM-Wire s memorijom poboljšana inačica legure Gold-Wire i konvencionalne legure Ni-Ti. No, duljine separiranih fragmenata pokazale su značajne razlike.

\section{Zaključak}

Sustavi izrađeni od legure CM-Wire (Hyflex EDM i Neoniti) bili su bolji od ostalih sustava kad je riječ o cikličkom zamoru. Kad je pak riječ o duljini separiranih fragmenata, F360 (konvencionalna legura Ni-Ti) i Reciproc (legura M-Wire) imali su dulje fragmente.

\section{Zahvala}

Autori zahvaljuju tvrtkama Komet Dental, EndovationsFKG Dentaire, Coltene, Dentsply Maillefer, Neolix, VDW i Micro-Mega na osiguranim instrumentima.

\section{Sukob interesa}

Autori izjavljuju da nisu bili u sukobu interesa.

\section{Sažetak}

Cilj: Ovo istraživanje temelji se na nultoj hipotezi kojom se tvrdi da nema znatnih razlika u otpornosti kad je riječ o cikličkom zamoru i duljini odlomljenih fragmenata između iglica veličine 25 u sustavima F360, F6 SkyTaper, Hyfelx EDM, iRace, Neoniti, One Shape Protaper Next, Reciproc, Revo-S te Wave One Gold. Materijali i metode: Odabrano je 300 novih iglica veličine 25 iz navedenih sustava $(n=30)$. Instrumente je pokretao endodontski motor X-Smart Plus s brzinom i okretnim momentom koje je preporučio proizvođač, bili su pričvršćeni u škripcu i pasivno prilagođeni bez pritiska u čeličnom bloku. Vrijeme potrebno da instrumenti puknu mjereno je u sekundama. Broj ciklusa zamora izračunat je kao otpor (s) x brzina/60. Duljina frakturiranih segmenata mjerena je digitalnom Vernierovom pomičnom mjerkom. Statistička analiza obavljena je programom SPSS 18 na razini 95-postotnog intervala pouzdanosti s pomoću Levenova testa usporedbe varijanci, Welchova testa usporedbe srednjih vrijednosti te Games-Howellova testa za otkrivanje razlika između skupina. Rezultati: Levenovim testom nisu otkrivene iste varijance $(P<0,05)$, Welchov test $(P<0,05)$ pokazao je statistički značajne razlike u cikličkom zamoru i za duljine frakturiranih segmenata. Games-Howellovim testom $(P<0,05)$ dokazana je statistička značajnost u višestrukim usporedbama $(P<0,05)$. Zaključak: Sustavi s CM žicom (Hyflex EDM i Neoniti) pokazali su se boljima u otpornosti na ciklički zamor od ostalih sustava. Kad je riječ o duljini frakturiranih segmenata, F360 (konvencionalna legura Ni-Ti) i Reciproc (M žica) bili su bolji u otpornosti.
Zaprimljen: 30. lipnja 2018 Prihvaćen: 12. prosinca 2018.

Adresa za dopisivanje Jorge Rubio Catholic University of Valencia Department of Endodontics and Restorative Dentistry jorrucli@hotmail.com

Ključne riječi

brzo rotirajuća stomatološka oprema; zamor; zakretni moment

\section{References}

1. Ankrum MT, Hartwell GR, Truitt JE. K3 Endo, ProTaper, and ProFile systems: breakage and distortion in severely curved roots of molars. J Endod. 2004 Apr;30(4):234-7.

2. Shen Y, Zhou HM, Zheng YF, Peng B, Haapasalo M. Current challenges and concepts of the thermomechanical treatment of nickel-titanium instruments. J Endod. 2013 Feb;39(2):163-72.

3. Peters OA. Current challenges and concepts in the preparation of root canal systems: A review. J Endod. 2004 Aug;30(8):559-67.

4. Mayer BE, Peters OA, Barbakow F. Effects of rotary instruments and ultrasonic irrigation on debris and smear layer scores: A scanning electron microscopic study. Int Endod J. 2002 Jul;35(7):582-9.

5. Pedullà E, Plotino G, Grande NM, Pappalardo A, Rapisarda E. Cyclic fatigue resistance of four nickel-titanium rotary instruments: a comparative study. Ann Stomatol (Roma). 2012;3(2):59-63.

6. Dhingra A, Ruhal N, Miglani A. Evaluation of single file systems Reciproc, One Shape, and Wave One using Cone Beam Com- puted Tomography -an in vitro study. J Clin Diagn Res. 2015 Apr;9(4):ZC30-4.

7. Capar ID, Ertas H, Ok E, Arslan H, Ertas ET. Comparative study of different novel nickel-titanium rotary systems for root canal preparation in severely curved root canals. J Endod. 2014 Jun;40(6):852-6.

8. Gambarini G, Grande NM, Plotino G, Somma F, Garala M, De Luca $M$, et al. Fatigue resistance of engine- driven rotary nickel-titanium instruments produced by new manufacturing methods. J Endod. 2008 Aug;34(8):1003-5.

9. $\mathrm{Xu} \mathrm{X}$, Eng M, Zheng Y, Eng D. Comparative study of torsional and bending properties for six models of nickel-titanium root canal instruments with different cross-sections. J Endod. 2006 Apr;32(4):372-5.

10. Franco V, Fabiani C, Taschieri S, Malentacca A, Bortolin M, Del Fabbro M. Investigation on the shaping ability of nickel-titani- 
um files when used with a reciprocating motion. J Endod. 2011 Oct;37(10):1398-401.

11. Wan J, Rasimick BJ, Musikant BL, Deutsch AS. A comparison of cyclic fatigue resistance in reciprocating and rotary nickel-titanium instruments. Aust Endod J. 2011 Dec;37(3):122-7.

12. De-Deus G, Moreira EJ, Lopes HP, Elias CN. Extended cyclic fatigue life of F2 ProTaper instruments used in reciprocating movement. Int Endod J. 2010 Dec;43(12):1063-8.

13. Yared G. Canal preparation using only one Ni-Ti rotary instrument: Preliminary observations. Int Endod J. 2008 Apr;41(4):339-44.

14. Berutti E, Paolino DS, Chiandussi G, Alovisi M, Cantatore G, Castellucci A, et al. Root canal anatomy preservation of WaveOne reciprocating files with or without glide path. J Endod. 2012 Jan;38(1):101-4.

15. Plotino G, Grande NM, Testarelli L, Gambarini G. Cyclic fatigue of Reciproc and WaveOne reciprocating instruments. Int Endod J. 2012 Jul;45(7):614-8.

16. Kosti E, Zinelis S, Molyvdas I, Lambrianidis T. Effect of root canal curvature on the failure incidence of ProFile rotary Ni-Ti endodontic instruments. Int Endod J. 2011 Oct;44(10):917-25.

17. Gambarini G, Miccoli G, Seracchiani M, Morese A, Piasecki L, Gaimari G, et al. Fatigue resistance of new and used nickel-titanium rotary instruments: a comparative study. Clin Ter. 2018 MayJun;169(3):e96-e101.

18. Champa C, Divya V, Srirekha A, Karale R, Shetty A, Sadashiva P. An analysis of cyclic fatigue resistance of reciprocating instruments in different canal curvatures after immersion in sodium hypochlorite and autoclaving: an in vitro study. J Conserv Dent. 2017 May-Jun;20(3):194-198.

19. Troian CH, So MVR, Figueiredo JAP, Oliveira EPM. Deformation and fracture of RaCe and $\mathrm{K} 3$ endodontic instruments according to the number of uses. Int Endod J. 2006 Aug;39(8):616-25.

20. Zbang L, L-Hong-xia, Zhou-Xd, Hong Tan, Huang-Dm The Shaping Effect of the combination of Two Rotary Nickel-Titanium Instruments in simulated S-shaped Canals. J Endod. 2008 Apr;34(4):456-8.ž

21. Goldberg M, Dahan S, Machtou P. Centering ability and influence of experience when using Wave One single-file technique in simulated Canals. Int J Dent. 2012;2012:206321.
22. Goldberg F, Araujo JA. Comparison of three instruments in the preparation of curved canals. Endod Dent Traumatol. 1997 Dec;13(6):265-8.

23. Elsaka SE, Elnaghy AM. Cyclic fatigue resistance of One Shape and Wave One instruments using different angles of curvature. Dent Mater J. 2015;34(3):358-63.

24. Zhang EW, Cheung GS, Zheng YF. A mathematical model for describing the mechanical behaviour of root canal instruments. Int Endod J. 2011 Jan;44(1):72-6.

25. Viana AC, Chaves Craveiro de Melo M, Guiomar de Azevedo Bahia M, Lopes Buono VT. Relationship between flexibility and physical, chemical, and geometric characteristics of rotary nickel-titanium instruments. Oral Surg Oral Med Oral Pathol Oral Radiol Endod. 2010 Oct;110(4):527-33.

26. Aminsobhani M, Meraji N, Sadri E. Comparison of cyclic fatigue resistance of five nickel titanium rotary file systems with different manufacturing techniques. J Dent (Tehran). 2015 Sep;12(9):63646.

27. Kaval ME, Capar ID, Ertas H, Sen BH. Comparative evaluation of cyclic fatigue resistance of four different nickel-titanium rotary files with different cross-sectional designs and alloy properties. Clin Oral Investig. 2017 Jun;21(5):1527-1530.

28. Pedullà E, Lo Savio F, Boninelli S, Plotino G, Grande NM, La Rosa $\mathrm{G}$, et al. Torsional and cyclic fatigue resistance of a new nickel-titanium instrument manufactured by electrical discharge machining. J Endod. 2016 Jan;42(1):156-9.

29. Ersoy I, Kol E, Demirhan Uygun A, Tanriver M, Seckin F. Comparison of cyclic fatigue resistance between different NiTi instruments with 4\% taper. Microsc Res Tech. 2016 May;79(5):345-8.

30. Topçuoğlu HS, Düzgün S, Akti A, Topçuoğlu G. Laboratory comparison of cyclic fatigue resistance of Wave One Gold, Reciproc and Wave One files in Canals with a double curvature. Int Endod J. 2017 Jul;50(7):713-717.

31. Keskin C, Inan U, Demiral M, Keleş A. Cyclic fatigue resistance of Reciproc Blue, Reciproc, and WaveOne Gold reciprocating instruments. J Endod. 2017 Aug;43(8):1360-1363.

32. Gündoğar M, Özyürek T. Cyclic fatigue resistance of OneShape, Hyflex EDM, WaveOne Gold, and Reciproc Blue nickel-titanium instruments. J Endod. 2017 Jul;43(7):1192-1196. 